\title{
Device Modeling and Measurement for RF Systems
}

\author{
Franz Sischka \\ Hewlett-Packard Company,Boeblingen Germany
}

\begin{abstract}
The trend to higher integration and higher speed challenges modelling engineers to develop accurate device models up to tens of Gigahertz. An absolute prerequisite for achieving this goal are accurate RF measurements, and correct de-embedding techniques. Without this, RF modelling can become quite time consuming, with a lot of guesswork and ad-hoc judgements. If, however, the underlying measurements are correct, and the models understood well, RF modelling can provide very accurate design kits.
\end{abstract}

Since the introduction of the first integrated circuit, consisting of a resistor, a capacitor and a resistor by Kilby in 1958, the number of devices per IC increased steadily. As a consequence, the size of integrated transistors increased as well. Since the early 1970ties, the gate diameter of MOS transistors has decreased by roughly a factor of 100 , see fig.1. This implies that the gate area has been reduced by a factor of $100_{-}=10000$. Since the operating frequency of such transistors is depending mainly on its capacitances, it becomes obvious that the cut-off frequency of modern MOS transistors can be far in the Gigahertz range, see fig. 2. 
min Gate size (um)

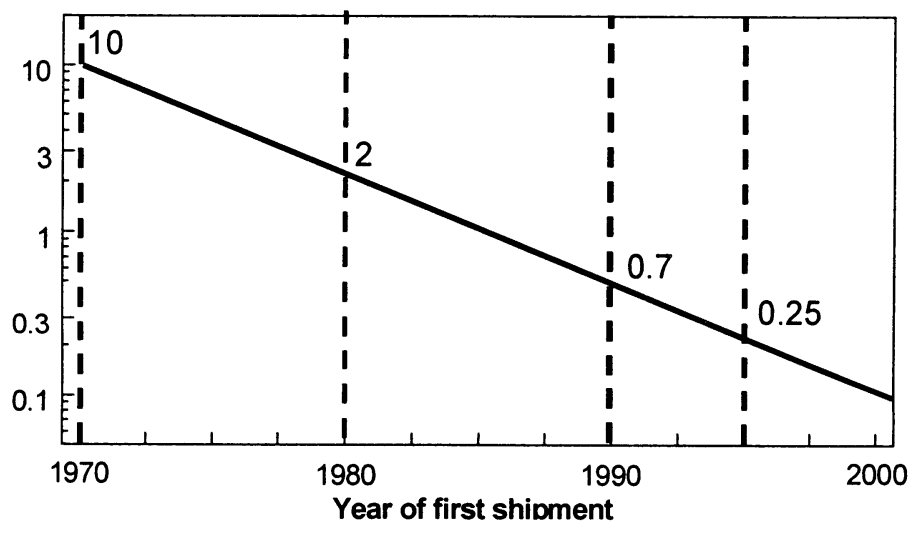

Figure 1: Minimum MOS Circuit Feature Size

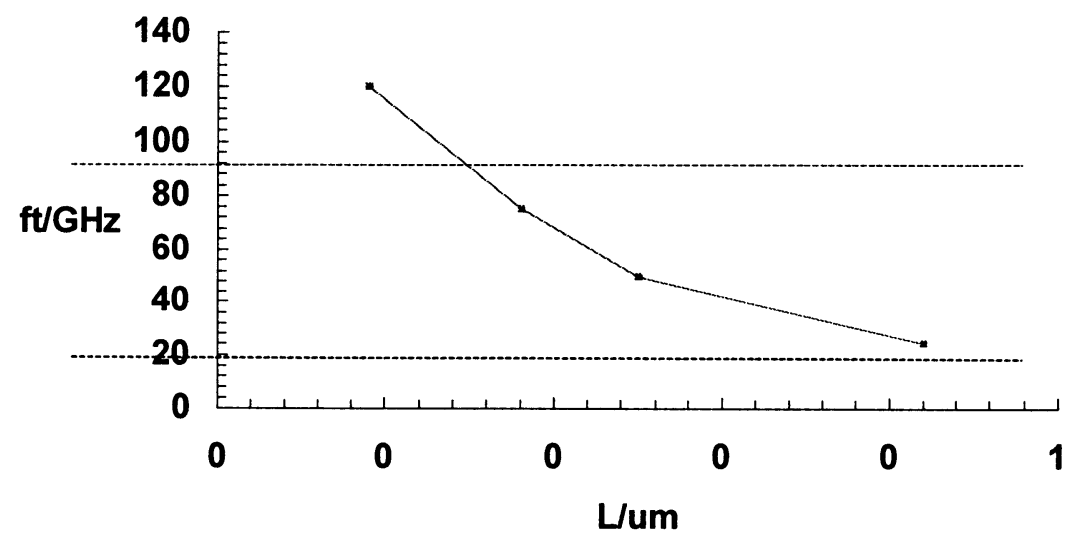

Figure 2: Silicon Transit Frequency Evolution

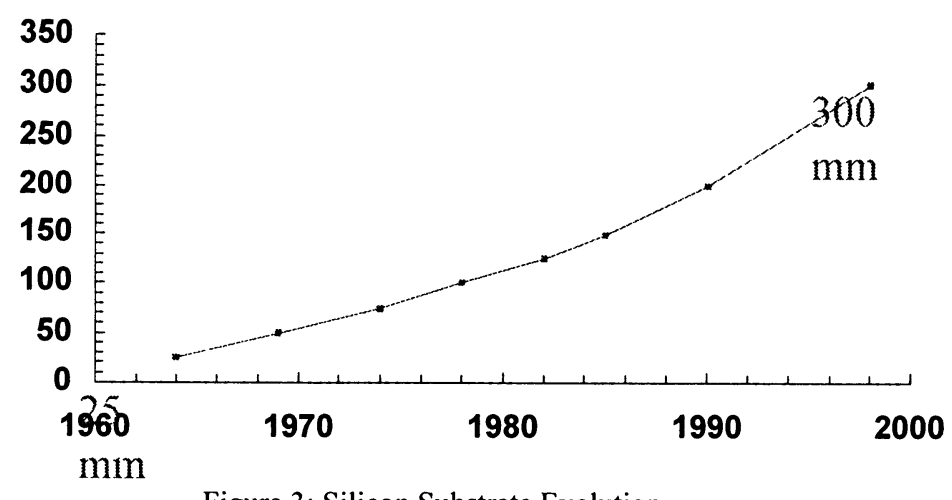

Figure 3: Silicon Substrate Evolution 
The interesting effect of this technology evolution is that the silicon wafer diameters increased during this time also drastically (fig.3), what allows today the development of sophisticated high-frequency VLSI chips for and affordable price.

This means: with increasing operating speed, cheaper manufacturing cost, fast turn-around and time-to-market, today's designs become more dependent on good device models. And, as can be seen in figure 4, device models are the base of the complete system design. For the digital design parts, relatively simple yet accurate enough time-dependent models can be developed, which describe basically the delay time of an electrical component, and its input and output characteristics. If, however, the design includes also analogue parts, like RF amplifiers, mixers etc., the weakest part of the design chain becomes the model of the analogue circuit, consisting of transistors and passive components. And, thus, the whole design becomes dependent on good models for these components.

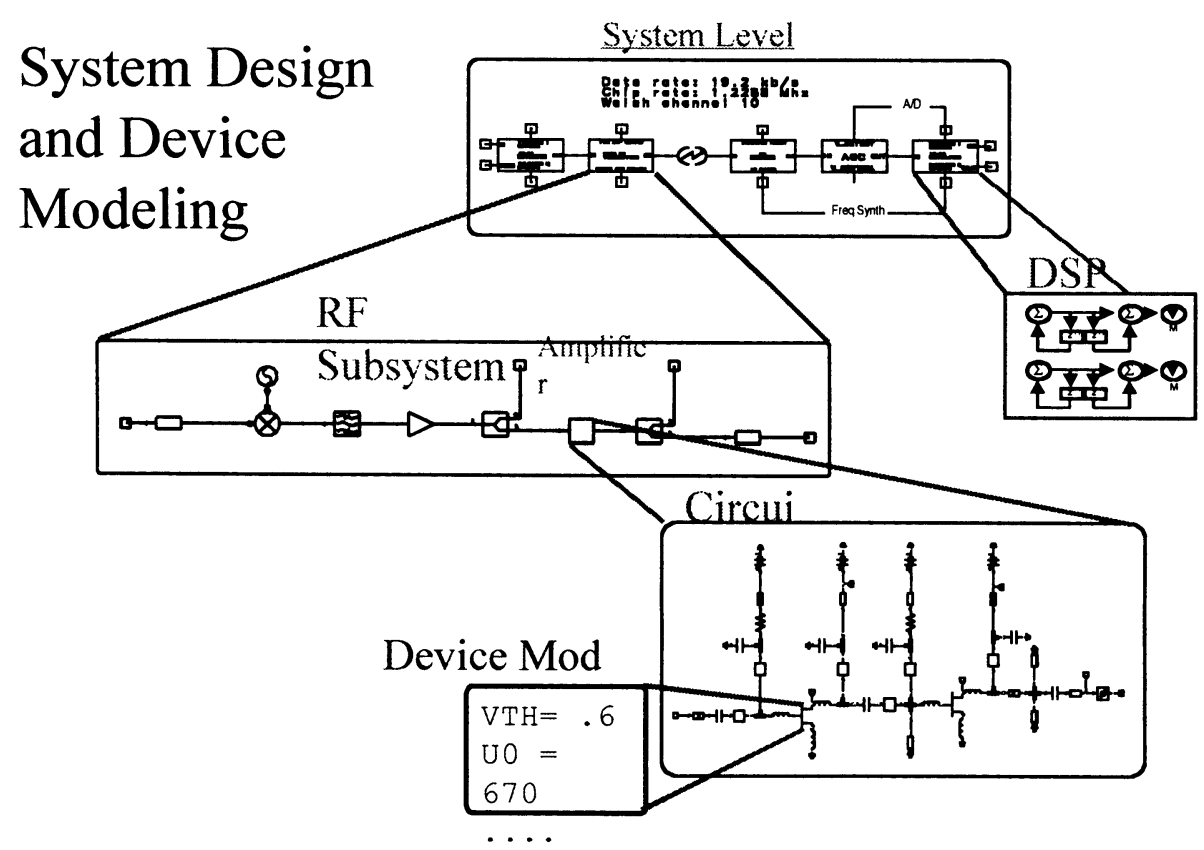

Figure 4: System Design and Device Modelling

In the simplest case, for an ideal ohmic resistor, the device model consists of a single formula, the well-known Ohmic law. And the model parameter is the Ohmic value $\mathrm{R}$, see fig. 5. In order to extract its parameter 
value, a current is applied to the resistor pins and the voltage drop measured. From a plot of $v$ over $i$, the calculated slope is equal to the value of $R$.

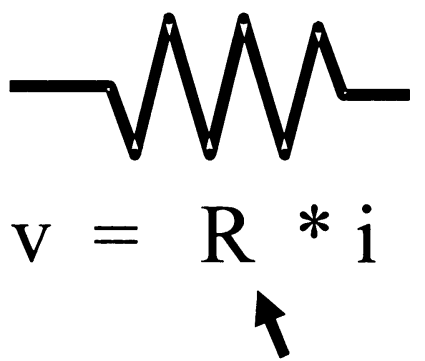

model parameter $\mathrm{R}$

.. is the slope of the resistance curve $\mathrm{v}(\mathrm{i})$

Figure 5: The Device Model of an Ideal Ohmic Resistor

MODEL TYPES:

EXAMPLE:

discrete models:

physical models

empirical models

individual transistors, diodes,
ideal passive components

tabular models

lumped, composed models: sub-circuit models

S-parameter 2-port blocks
4
.
transistors including parasitics,
e.g. npn with paras. pnp
spiral inductors, varactor diodes, mim capacitors, resistors on silicon
packages

if supported by the simulator

Figure 6: Model Types for Electronic Components

For more complex components, the models become more complex too. Figure 6 gives a rough overview. For individual transistors, diodes and ideal passive components, discrete models are available. They can be distinguished between physical models, empirical models and tabular models. Physical models try to include as much as possible equations from device physics. An example is the diode current $i=I S^{*} \exp (v / v t)$, characterised by the saturation current IS and the temperature voltage vt. While IS is a model parameter, vt is calculated by the simulator out of the 
desired device simulation temperature. This physical based type of models can be very accurate for low frequencies, but may become very complex for high frequencies. For this reason, their implementation into simulators is generally a simplification. And this is exactly what causes the problems for RF modelling. The simplification is required in order to allow circuit simulators evaluate the performance of a circuit of thousands of individual circuit components. By this way, the empirical models, based on mathematical equation fitting, come into play. They can also be combined with physical equations. The drawback is, however, that the physical background of a model is lost, and the interpretation of the model parameters becomes doubtful.

On the other hand, tabular models which consist of measured RF data at many bias points, which are interpolated during the simulation, can be a way out of this dilemma. However, they are usually simulator dependent and their migration to another simulator can be pretty difficult.

A commonly used method is to combine discrete models with subcircuits, when it comes to RF modelling. This way, it is possible to model bipolar transistors including parasitics (e.g. a npn transistor with a parasitic pnp), and MOS transistors using for example the BSIM3 model up to $30 \mathrm{GHz}$ and more. In the later case, an additional gate resistor and a small R-L circuitry between drain, bulk and source makes physical sense and helps in achieving this modelling result. Back to the firstly mentioned passive components, i.e. resistors on silicon, spiral inductors, varactor diodes mim capacitors etc., their RF characteristics can be modelled very accurately when using sub-circuits consisting of individual, lumped RLC components. As a side effect, such sub-circuits are simulator independent, and thus can be ported easily to another design environment. This kind of modelling can also be applied to package modelling, a domain which will become more and more important.

Some major RF simulators also allow the import of measured Sparameters for given bias points. This way, cumbersome large sub-circuits which are often required for geometrically big components like packages can be simplified drastically. However, a drawback is the already mentioned reduced portability to other simulators and also the fact that problems with the measurement can easily be hidden behind the S-parameter blocks. Doing modelling the 'conventional' way will in this case lead to either unphysical model parameters or bad fitting performance of the model, and will therefore be detected. Also, a frequency-dependent model parameter in the case of sub-circuit modelling is mostly a hint for a need to improve the equivalent 
sub-circuit. An experienced modelling engineer can read out of this dependency the required sub-circuit enhancement.

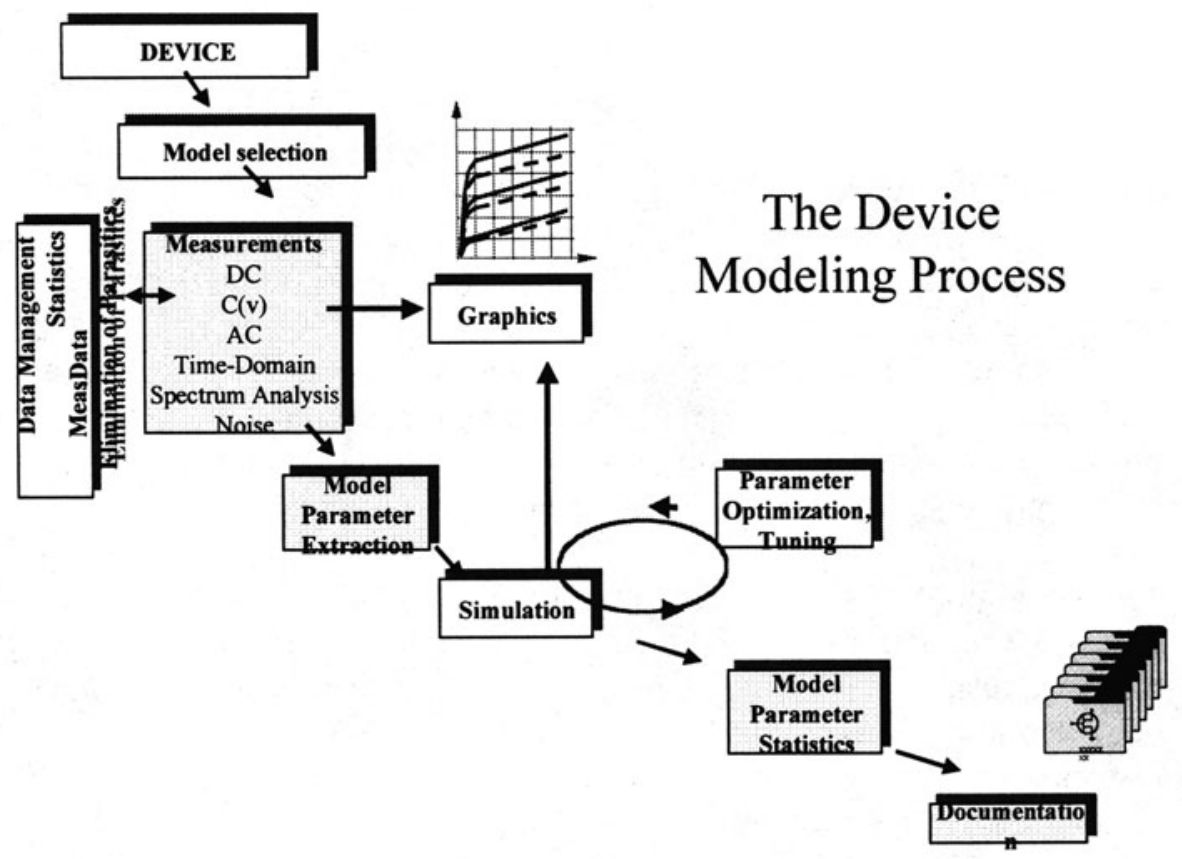

Figure 7: The Device Modelling Process

Figure 7 depicts the global device modelling process. For a given device, a model is selected first. The model equations, which are solved for the model parameters during model parameter extraction, give a clear indication about what kind of measurements and what type of stimulus sweeps are required for characterisation. During the parameter extraction process, the selected model will fit more and more precisely the measured device. This is done by starting with so-called default model parameters, not to be confused with 'typical' parameters. These default parameter values are usually set so that they behave neutral. As an example, a default parameter for a capacitor is zero. In the case of a bipolar transistor, the first model parameters extracted are the DC parasitics RE and RC, followed by the Early voltage and the current amplification beta. The other parameters are still set to default. The so far determined model is a simple Ebers-Moll model, with bias-independent beta, and no frequency dependencies. Step by step, more and more parameters are determined and the model becomes more and more precise with respect to DC and finally RF. Figure 8 depicts this scheme. For a general transistor modelling, the DC performance with respect to its input 
and output characteristics as well as its transfer function is done first, followed by the so-called CV modelling, i.e. the characterisation of the depletion capacitances. Finally, the S-parameters are measured and modelled by the transit time parameters.

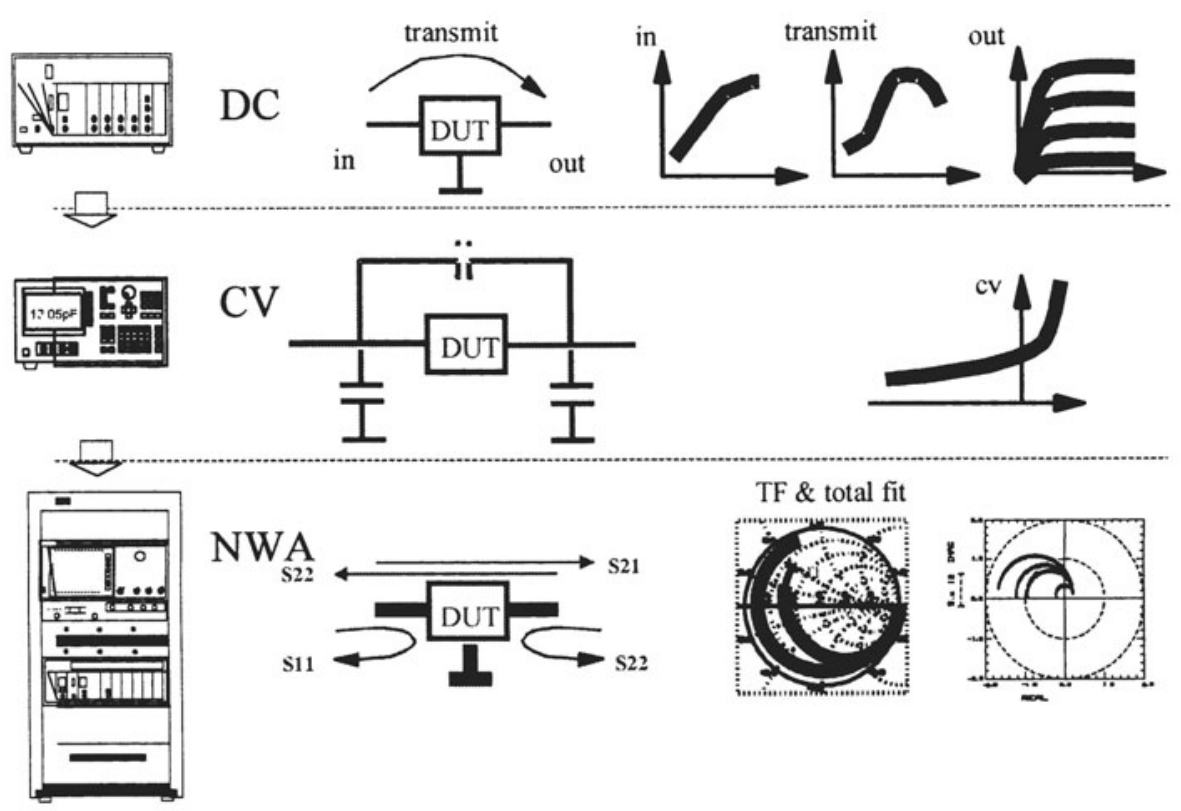

Figure 8: Basic Device Modelling Measurements

Before leading over to the pure RF modelling aspects, figure 9 shows the dilemma to identify the right device for modelling. This means that before an individual component is 'just' selected to serve as a model, it must be assured that this component represents a typical device. Therefore, before doing modelling, some statistical analysis has to be applied to the measured data in all measurement ranges. This is called measurement data management. 


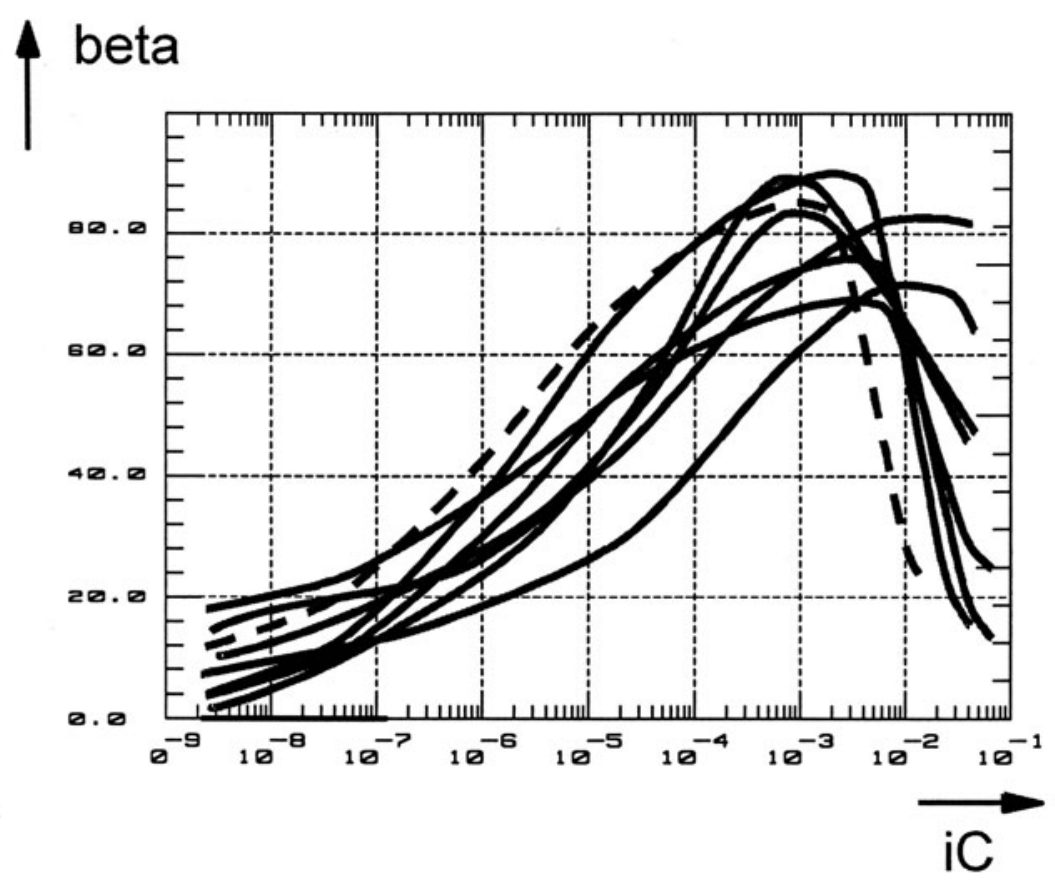

Figure 9: Measured beta traces of bipolar transistors on a wafer

It is also recommendable to perform a measurement for as many DC sweeps as possible and a full frequency S-parameter sweep with as many as possible DC bias conditions. Although this 'over-measurement' may not be required for modelling itself, it is very valuable when comparing the set of simulated data after the model has been established with these many measurement data and to analyse the percentage error over all the bias conditions. Only if this is performed, the developed model can guarantee the desired model accuracy.

Provided the model obtained parameter set as well as the possibly required sub-circuit makes physical sense, the model can be assumed to be accurate also for those operating conditions which may not have been measured for modelling.

\section{$\underline{\mathrm{RF} \text { modelling aspects: }}$}

Like sketched in figure 10, S-parameters may easiest be compared to a beam of light, which hits a pair of spectacles. A part of the incident beam is reflected, and the other part is transmitted. With S-parameters, the device under test is tested similarily: first its input characteristics and transfer 
characteristics from port 1 to port 2 (output) is characterised, and then the same is done backwards. Such a way, it's RF characteristics is completely evaluated.
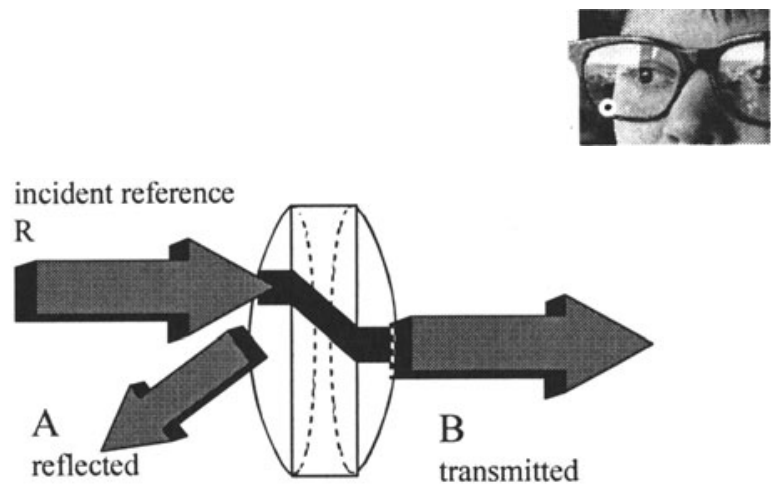

Figure 10: S-Parameter Analogy

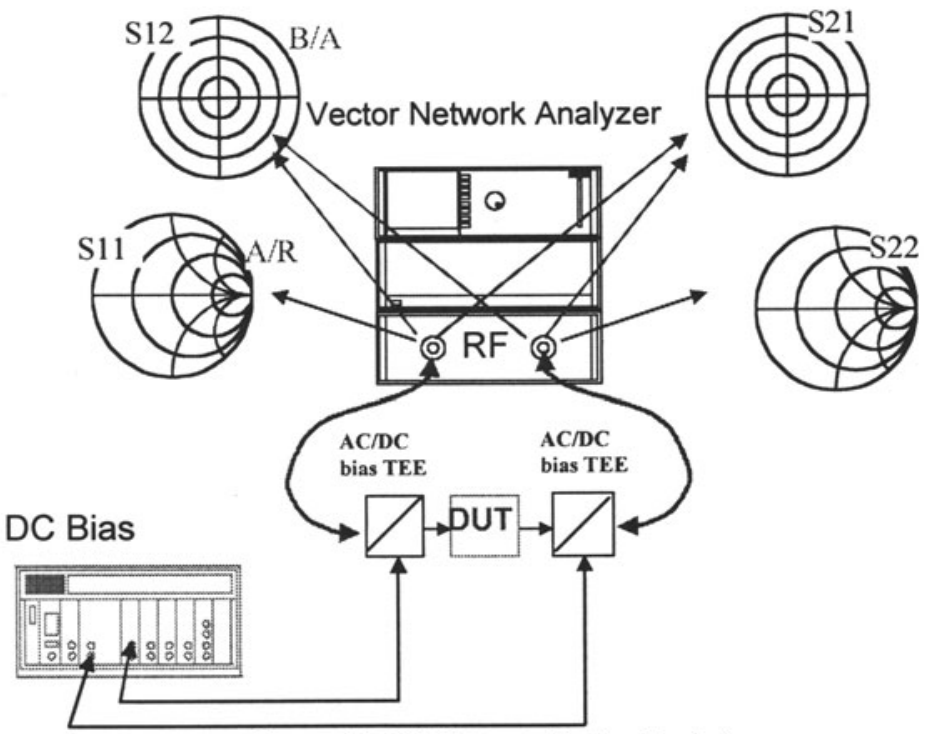

Figure 11: RF Characterisation Techniques

Figure 11 depicts the required measurement setup. The DC and AC signal is combined by the AC/DC bias TEEs. It must be assured that there is no overdrive of the transistor under test, because the network analyzer is a linear system. Therefore, the forward transfer curve must be measured with a much smaller AC signal than the reverse case. From the input reflections of the DUT, S11 is measured as the ratio of incident power to reflected power, i.e. $\mathrm{A} / \mathrm{R}$ of figure 10 . The transfer characteristics is obtained by the network analyzer by calculating the ratio $\mathrm{B} / \mathrm{A}$. A second measurement in reverse 
mode concludes the DUT characterisation. It should be mentioned that for this kind of measurements, special test structures are required. An example is given in fig. 12. Because of the RF frequencies, the test pins also need to include a proper ground connection in order to connect the ground shield of the RF cables properly and with an extremely short way to the DUT. If this is not done properly, self-oscillation of the DUT may occur and the modelling will fail.

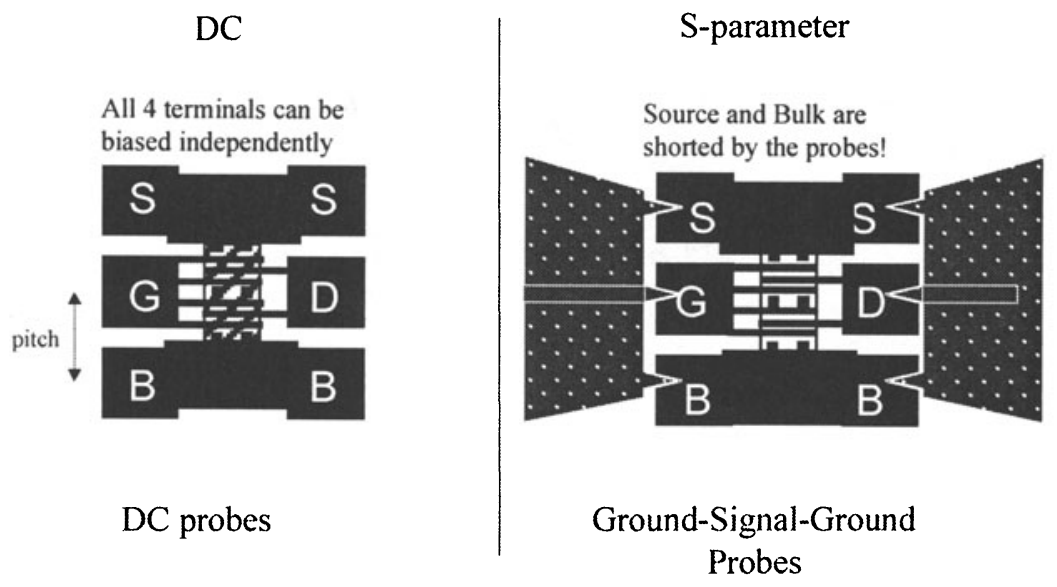

Figure 12: RF modelling measurements require specific test device layout

It is also important to note that the starting points of the S-parameter traces for low frequencies are completely determined by the DC characteristics. For the modelling, this means that the starting points of the simulated traces are in the same way determined by the DC model parameters. The RF transistor parameters only influence the further trace of the S-parameter curves. Therefore, in order to obtain an accurate RF large signal model, a proper DC modelling is a must.

Another prerequisite for good RF models is an accurate de-embedding of the RF parasitics. Firstly, they stem from the network analyzer (cross-talk between the ports, frequency dependence of the port amplifiers etc.), and the delay of the measurement cables. These effects, however, can be eliminated by proper network analyzer calibration. The remaining parasitics, like the on-chip test pad capacitances, as well as parasitic inductances for frequencies above about $10 \mathrm{GHz}$, need to be eliminated by de-embedding. Otherwise, the measured device characteristics is not that of the very inner device alone, but also includes the overlying parasitics. Even if the model fits well, its parameters are physically incorrect, because they reflect both, the very inner device plus the outer parasitics. Since these parasitics will not 
exist in the later RF design (e.g. the test pads), they need to be stripped off. This procedure is called de-embedding. In most cases, this is done by twoport matrix manipulations. As an example, capacitances, representing basically the test pads, and which are measurable with an OPEN dummy, can be subtracted by subtracting their $\mathrm{Y}$ matrix from the total measurement. Consequently, a SHORT dummy measurement, de-embedded first (!) from its outer test pads, represents the delay from the pin contacts to the inner device. Its parasitics can be eliminated by subtracting the SHORT dummy Z matrix from the total measurement. As stated before, the SHORT has to be de-embedded from the outer OPEN parasitics first.

Figure 13 shows the effect of overlying parasitics for a cut-off frequency measurement, and figure 14 sketches the de-embedding process and the required twoport matrix manipulations.

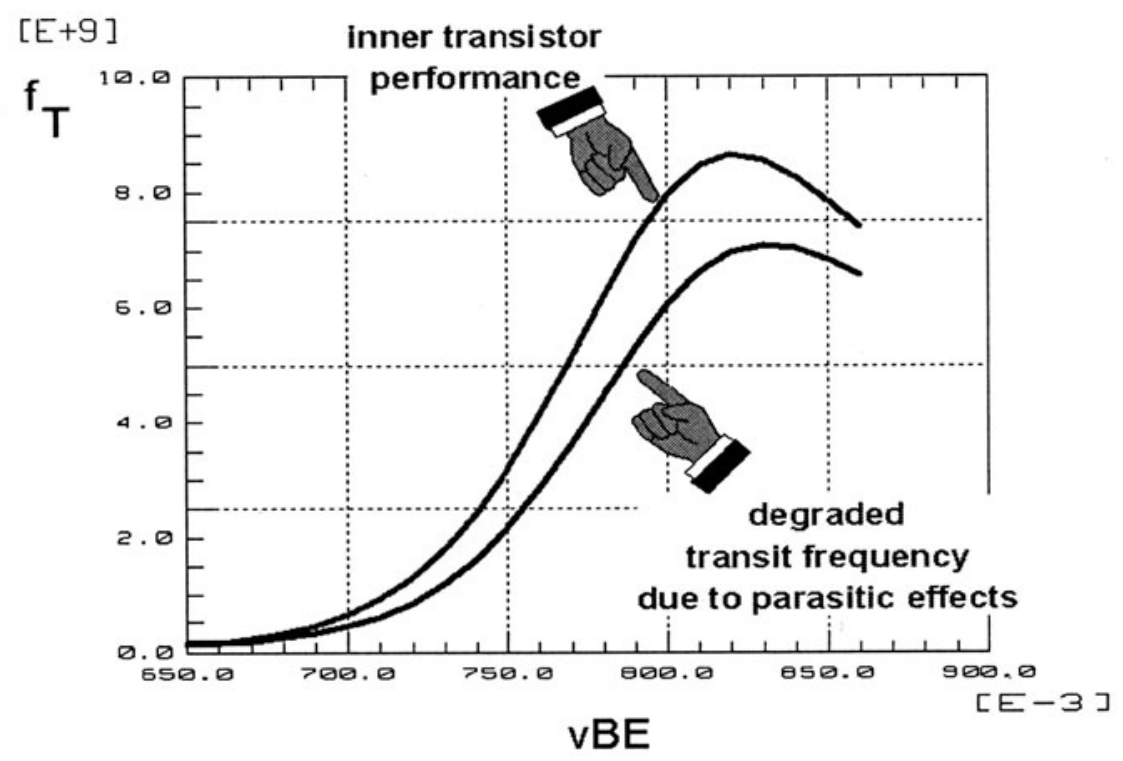

Figure 13: De-embedding Means Off-Stripping of Overlaying, Measurement Related Parasitic Effects. 


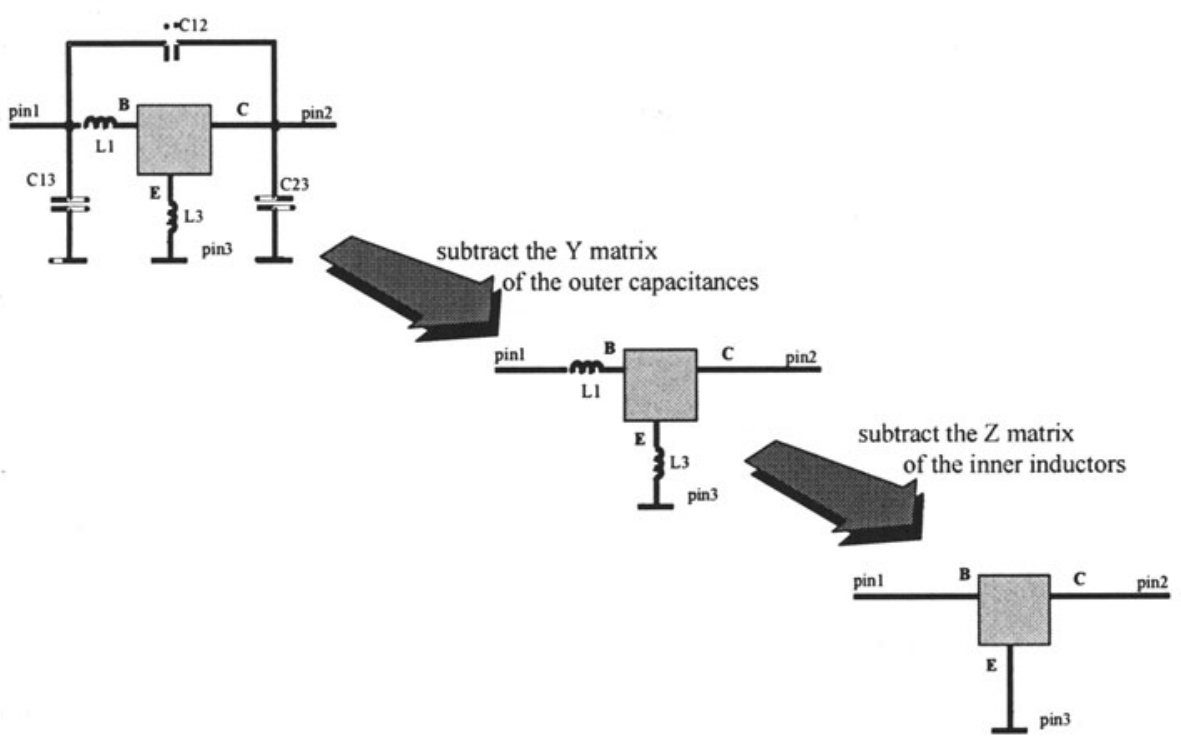

Figure 14: The De-embedding Procedure

As mentioned before, after the de-embedding, the modelling of the RF performance of transistors is basically the correct determination of the transit time model parameters. If the model exhibits some RF model limitations, additional small sub-circuits can be used to match the frequencies in the higher $R F$ range.

If only a transistor model for a fixed DC operating point is required, a small signal model might be sufficient. Figure 15 gives an example. The components of the model branches are defined by evaluating the deembedded $\mathrm{Y}$ matrix of the inner transistor and by displaying the inverse in the complex impedance plain. Such a way, the required branch structure and the parameter values of its components become clearly visible and can be modelled accordingly. 


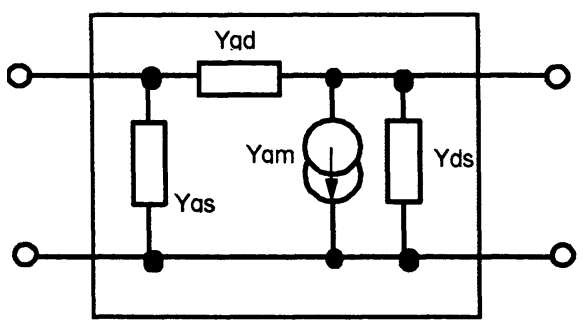

$$
\left(\begin{array}{cc}
Y 11 & Y 12 \\
Y 21 & Y 22
\end{array}\right)=\left(\begin{array}{cc}
Y g s+Y g d & -Y g d \\
Y g m-Y g d & Y d s+Y g d
\end{array}\right)
$$

Solved for the comnlex conductances:

$$
\begin{aligned}
& Y g d=-Y 12 \\
& Y g s=Y 11+Y 12 \\
& Y d s=Y 22+Y 12 \\
& Y g m=Y 21-Y 12=g m \times \exp (-j \omega T A U)
\end{aligned}
$$

Figure 15: Operating Point RF Modelling for Transistors

When it comes to passive components modelling, be it on-chip or packaged, the standard models of inductors, capacitors and resistors are not sufficient for RF. Therefore, sub-circuit modelling or the development of custom RF models for those components is required. Good RF simulators allow such model development. However, migration of the model to other types of simulators will become difficult. For passive components, the schematic and the parameter values are extracted again from the complex impedance plain.

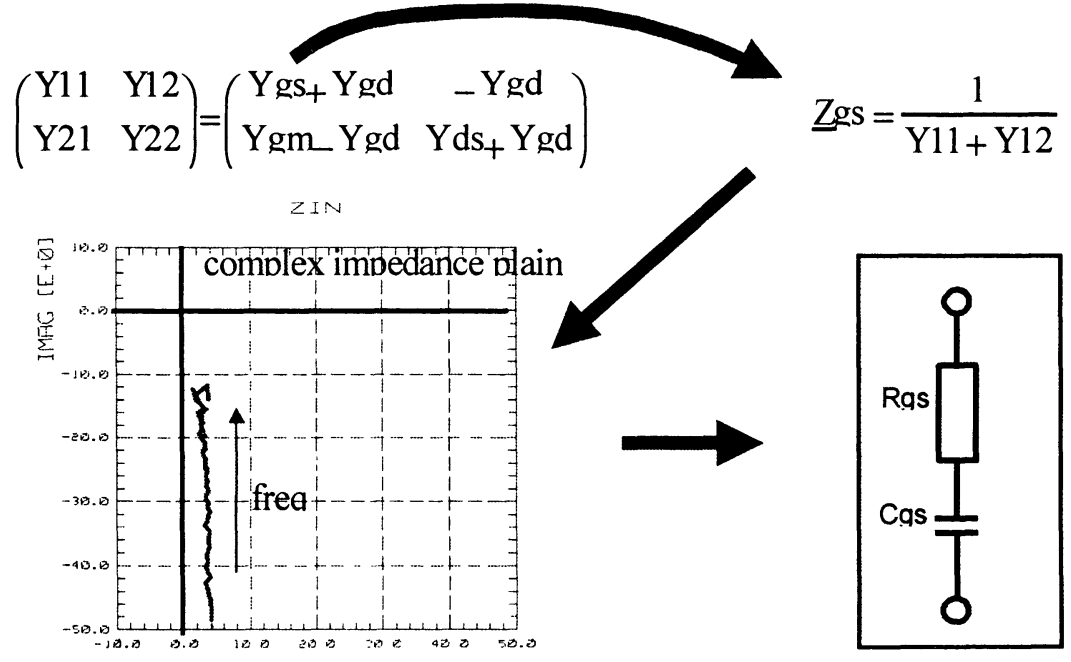

Figure 16: Developing the Schematic and Extracting the Component Parameter Values out of the Complex Impedance Plain 
Last not least, the noise performance needs to be modelled. For basically all transistor types, the $1 / \mathrm{f}$ noise model is included in the device models and the corresponding parameters can be extracted. Like with the RF modelling, an accurate measurement is a prerequisite for successful noise modelling. Some modern modelling tools also allow the modelling of the non-linear RF performance like $3 \mathrm{rd}$ order intersect and $1 \mathrm{~dB}$ compression point. Such type of modelling ensures the best model accuracy possible.

Conclusions:

Provided the RF measurements and the measurement data handling has been performed accurately, and the DC modelling is correct, experienced modelling engineers can generate accurate device models up to the high RF frequency range. A pre-requisite is good experience in RF measurement techniques and a flexible RF modelling tool, which supports the engineer with the development of a suitable RF model and the extraction of the corresponding model parameters. 